\title{
Experiencia de los cuidadores de enfermos crónicos en el hospital
}

\author{
Rosa Ma. Guadalupe Andrade Cepeda
}

RESUMEN

Objetivo: conocer las experiencias de cuidadores de enfermos crónicos en el hospital: Material y métodos: estudio cualitativo, fenomenológico con 16 cuidadores de pacientes hospitalizados en medicina interna de un hospital de Seguridad Social, participaron 13 mujeres y 3 hombres, de las mujeres el parentesco fue: esposas, hijas, hermana, todas amas de casa; de los hombres: esposo e hijos, se realizó a través de entrevistas a profundidad que fueron grabadas previo consentimiento informado, se realizó análisis de contenido y se proceso en el programa Atlas Ti 4.2. Resultados: predominaron los cuidadores del sexo femenino de mediana edad, amas de casa, que dedican 12 horas al cuidado, se presentaron diferencias en cuanto al significado del cuidado entre las esposas que lo ven como obligación y los hijos como responsabilidad o correspondencia, para estos cuidadores el hospital es un medio extraño; señalan que el cuidar implica satisfacción de necesidades básicas de seguridad y terapéuticas, que aprenden por ensayo y error, por la temporalidad se presentan repercusiones fisiológicas y psicológicas además reorganizan sus actividades cotidianas para poder cuidar. Conclusiones: la experiencia de cuidar en el hospital va desde cómo se visualiza el contexto del hospital como un medio desconocido hasta la organización de la vida cotidiana, sería importante incluirlo en los planes de atención al paciente y hacer visible la contribución.

Palabras clave: experiencia de cuidadores, cuidadores en el hospital.

\section{Caregivers' experience of the chronically ill in hospital}

\begin{abstract}
Objective: to know the experiences of caregivers of the chronically ill in hospital. Material and Methods: a qualitative, phenomenological study with 16 caregivers of hospitalized patients in internal medicine at Social Security, involving 13 women and 3 men; the relationship women was: wives, daughters, sisters, all housewives; the men, husband and son. Was conducted through in depth interviews that were recorded prior informed consent. Was analyzed for content and process in Atlas Ti 4.2. Results: the caregivers prevaleiled female middle-age housewives, spend twelve hours care, there were differences in the meaning of the between the wives as they see soon as an obligation and responsibility or correspondence, for these hospital care is half the strange report that caring involves meeting basic needs, security and therapy, they learn by trial and error, by the temporality effects are physiological and psychological well reorganize their daily activities to care. Conclusions: the experience of caring in the hospital is viewed as running form the hospital as a means unknown to the organization of very life to care, it is important to include in the plans for patient care and make visible the contribution.
\end{abstract}

Key words: experience of caregivers, caregivers in the hospital.

Correspondencia: Rosa Ma. Guadalupe Andrade Cepeda. Facultad de Enfermería de la Universidad Autónoma de San Luis Potosí. Niño Artillero \# 130 Zona Universitaria. E-mail: randrade@uaslp.mx

$\mathrm{E}$ n México el proceso de envejecimiento de la población ${ }^{1}$ aunado a la transición epidemiológica representa un incremento importante de enfermedades crónicas degenerativas $^{2}$ con problemas de dependencia y / o déficit funcional para desarrollar actividades de la vida diaria ${ }^{3}$, situación que se exacerba durante la enfermedad, ante este hecho la familia, es quien asume la responsabilidad del cuidado por ello se le reconoce como un agente cuidador natural.

Una de las funciones básicas de la familia es la formación, protección física y emocional de sus miembros, por lo que asume la responsabilidad de cuidar la salud y satisfacer sus necesidades ${ }^{4}$, por razones de enfermedad prodiga sus cuidados en el ámbito domiciliario o durante la hospitalización. En 
situación de hospitalización se requiere de la presencia de una persona que permanezca con el paciente durante su estancia, se convierte en un soporte fundamental para el cuidado, en este estudio a esa persona se le denominará cuidador informal el cual sin ser profesional proporciona la mayor parte de asistencia y apoyo diario, ayudando al desarrollo de las actividades de la vida diaria del enfermo, este tipo de cuidado se caracteriza por ser continuo y no remunerado ${ }^{5,6}$.

Las investigaciones al respecto ponen de manifiesto que el cuidado informal tradicionalmente recae en las mujeres, esposas, hijas o hermanas, que dejan hogar e hijos, señalando que los hombres tienen menos probabilidades de brindar cuidados, excepto cuando el familiar al que se cuida es un hijo o padre.

Estos cuidadores se encuentran presentes en cualquier hospital, público o privado, su permanencia contribuye a disminuir la incertidumbre causada por la normatividad de la institución que establece horario de actividades ${ }^{7}$, se ha referido que este tipo de cuidados representa una carga física y psíquica, que repercute de manera negativa en la calidad de vida del cuidador ${ }^{4,6,8,9}$.

Una vez que el enfermo ingresa al hospital la responsabilidad de los cuidados pasa de los familiares al personal, con lo que puede disminuir la carga, o bien en caso de cuidadores con experiencia continúan realizando el cuidado tal como el paciente está acostumbrado. Sin embargo, en hospitales la figura del cuidador informal no se considera necesaria y por lo tanto no está normado, su participación pasa desapercibida, no valorada, pues se considera que el objeto de los cuidados es sólo el paciente $^{10}$.

A partir de los últimos años la literatura ha evidenciado la importancia de los cuidadores sobre todo en el ámbito domiciliario, su participación ha sido estudiada desde varias perspectivas como identificación del perfil y repercusión en su salud por la sobrecarga de cuidado. En México no existen evidencias sobre el cuidador en el ámbito de hospitalización, por ello el estudio de este tema desde la orientación cualitativa permite explorar vivencias del cuidador de pacientes hospitalizados de este modo contextualizar el medio y conocer su posición en la adaptación de su vida para el cuidado, además posibilitará nuevas alternativas para hacer visible su participación en el cuidado, y coadyuvar esfuerzos para mejorar su calidad de vida y por ende la del receptor de sus cuidados.

\section{MATERIAL Y MÉTODOS}

Investigación con enfoque cualitativo, de carácter fenomenológico que se enfoca en determinados fenómenos tendientes a describir la experiencia humana, que enfatiza en los procesos de los significados y experiencias humanas, construidas de la realidad vivida por los cuidadores informales de pacientes hospitalizados ${ }^{11}$.

\section{Participantes en el estudio}

Para la selección de los participantes se consideraron los cuidadores de adultos mayores; clave para el objetivo del estudio, para lo cual se estableció coordinación con los jefes de piso de los servicio de medicina interna y cirugía de una institución de seguridad social, una vez identificado el cuidador se procedió a solicitar su consentimiento informado y establecer cita para realizar la entrevista.

Participantes: cuidadores informales 16 de los cuales 13 fueron mujeres y 3 hombres, de las mujeres 3 esposas, 9 hijas y una hermana, todas amas de casa; de los hombres 1 esposo y 2 hijos, de ocupación un maestro de primaria, 1 recolector de basura independiente, y otro jubilado; con edad promedio de 56.4 años; los días de cuidado fueron en promedio 10.1 (2 -30) y de 8 a 16 hs dedicadas a esta actividad. Los adultos mayores hospitalizados 8 eran hombres y 8 mujeres, con un promedio de edad 78.6 años (61-97), todos ellos con enfermedad crónica: 8 con complicaciones de diabetes, dos con EVC, 2 con problemas coronario, uno neumonía, uno deshidratación y anorexia y otro esclerosis lateral amniotrófica.

Procedimiento: la recolección de la información fue a través de entrevista semiestructurada, las cuales fueron grabadas con el consentimiento informado, cada entrevista duró de 30 a 45 minutos, todas se realizaron en el hospital, se grabaron y se transcribieron en su totalidad. El análisis de los datos fue a partir del análisis de contenido, se realizó codificación para obtener categorías y subcategorías.

\section{RESULTADOS}

En este estudio encontramos pacientes con problemas crónicos y por lo tanto con diversas hospitalizaciones; los cuidadores describen su experiencia desde el proceso del diagnóstico, emociones generadas por tener un familiar hospitalizado y como organizar su vida efectuar esa función. A partir del análisis la experiencia del cuidador está conformado por: significado del cuidado, hospital como contexto para el cuidado informal, tarea del cuidador en el hospital. La organización para el cuidado, peso y fortaleza para el cuidado.

\section{Significado del cuidado}

Desde el inicio de la enfermedad los cuidadores van construyendo su experiencia al lado de su familiar enfermo, el proceso diagnóstico es donde la reacción emocional y adaptación a la enfermedad, tienen diferentes connotaciones dependiendo si se es el cónyuge o hijo.

Los papeles desempeñados por los diferentes miembros de la familia, se derivan de una diferenciación sexual del trabajo al 
interior de esta, que como parte del proceso de socialización es internalizado, el papel masculino se mantiene preponderantemente como un ser instrumental y el femenino como más expresivo lo que ha llevado a la mujer a asumir la función del cuidado de esta manera las mujeres son las que se responsabilizan del cuidado y los hombres desempeñan un papel marginal ${ }^{12}$.

Es así como se establece la diferencia del porque cuida dependiendo del papel familiar, se trata de un acto personal reciproco, que supone ayuda, para el esposo el cuidar se convierte en una responsabilidad.

...tengo la responsabilidad desde que nos casamos ahí recibí la responsabilidad. Las esposas refieren que cuidan por obligación. Porque es mi esposo y yo tengo la obligación de cuidarlo y lo hago con gusto o también refieren que es por afecto y deber.

«....es mi esposo es la obligación, afecto, no es amor, así después de 58 años de casados no creo, es deber debo de cumplir hasta el día de que pueda».

Para los hijos el cuidar se convierte en responsabilidad sobre todo cuando los padres por edad y discapacidad ya no trabajan el cuidado visto como parte de sus deberes filiales².

«...porque es uno de su sangre, es una responsabilidad que uno tiene bajo sus padres»

También lo refieren como un acto de correspondencia.

«...nos toca como hijos cuidar a los padres y hay que estar al pendiente, cuando éramos chiquitos ellos nos cuidaron y atendieron ahora nos toca a nosotros»

El porqué realiza la función de cuidar, también se ve matizado de acuerdo al estado civil «...estoy soltera entonces yo siento que es mi obligación, si ella me cuido de chiquita pues ahora me toca a mi cuidarla»

Asimismo cuando la red de cuidadores es reducida es percibido como una obligación.

«...es mi papá; es mi obligación no hay nadie más que venga a cuidarlo, todavía hay que cuidarlo ni modo el ya esta viejito.»

\section{El hospital como contexto para el cuidador informal}

La inmersión en la vida y estructura hospitalaria genera un cambio profundo en la representación de papeles habituales, la hospitalización representa para el paciente y su cuidador un desafío a su capacidad de adaptación por estar inmerso en un medio extraño y con personas desconocidas ${ }^{7,13,14}$.

«...la primera vez no sabíamos y nos piden que ponga uno esto aqui y que valla y uno no sabe ni para donde»

Los cuidadores refieren que es un medio incomodo por el espacio.

«...no hay privacidad, siente uno como si se asfixiara quisiera tener el espacio que tengo en mi casa»

Las salas de hospitalización son para tres camas, y en la institución donde se realizó el estudio en los privados que son para una cama se ponen dos separado por un buró, entonces el espacio se reduce hasta para que las enfermeras realicen sus actividades el cuidador solo tiene una silla junto a la cama o en los pies de ella según sea el espacio, por ello el cuidador percibe esta situación y señala.

«...la relación se tensa al no tener espacio suficiente, las enfermeras dicén hágase para allá y ellas también se ponen nerviosas porque tienen muchos enfermos»

Como parte del contexto se señalan los olores y refieren que en donde estén siguen percibiendo el olor del hospital «...luego se va uno y queda así como que el olor del hospital pero llega uno se baña y se cambia, si se penetra uno» E3

Otra característica del hospital según los cuidadores del turno nocturno es sobre lo que escuchan

«...oía cuando fallecían las personas o, cuando andan las enfermeras dándoles los medicamentos y algunos enfermos que no se dejaban poner algún medicamento»

Tarea del cuidador en el hospital

La participación en el cuidado es básica al realizar actividades tendientes a satisfacer las necesidades primarias.

«...me dice la señorita ayúdeme a cambiarlo, voltearlo y me dice no sea malita póngale el oxígeno y así cualquier cosa, darle de comer por la sonda»

«...Lo volteamos ya sea para allá, para acá para evitar lo menos que puedan aspirarlo, le damos masaje»

También satisfacen necesidades de seguridad, colaboración en las medidas terapéuticas, de acuerdo al nivel de dependencia.

«...estar al pendiente de que no se fuera a quitar el suero o sus medicamentos»

«...cuidándolo, amarrándolo porque se suelta y se jala la sonda si lo dejamos sólo»

Sin embargo, refieren tener escaso apoyo por parte de enfermería por lo tanto consideran que estas actividades las realizan sin conocimientos ${ }^{15,16}$. 
«...la señorita a veces me dice que le ayude o que haga alguna cosa pero a mi me da miedo y me dicen como, es que a ellas (enfermeras) también...»

Van aprendiendo a cuidar por ensayo y error o bien viendo de los demás

«... primero uno no sabe cómo y después aprende de los demás; uno va agarrando confianza a los médicos y enfermeras púes a todo..»

En ocasiones el personal de enfermería no permite que el cuidador permanezca cuando están realizando algún procedimiento como el baño, que cuando sea dado de alta el cuidador lo tendrá que realizar.

«...cuando lo bañan a veces si nos dejan algunas enfermeras estar aquí para que veamos, otras nos dicen no, nos sacan»

Organización para el cuidado

El cuidado a un familiar hospitalizado implica cuidado las $24 \mathrm{hs}$ del día, esto hace necesario organización para desempeñar la función de cuidador y dividir las 24 hs, para determinar las horas de cuidado y actividades propias de cada cuidador.

La organización en turnos de cuidado que en esto depende si es hombre o mujer o bien del estado civil. En este punto encontramos que cuando es la esposa esta cuida de día y por la noche la auxilian los hijos.

«...nada mas de día estoy yo, mis hijos de noche porque tienen que trabajar, si yo aguantará estaría día y noche con tal de que ellos no se fueran desvelados a trabajar»

Cuando se trata de cuidar a la mamá las hijas asumen el cuidado y depende del número como establecen los horarios

«...entre las 5 hijas estamos aquí, se queda una toda la noche hasta las 6 de la mañana, llega otra y se queda hasta las 2 de la tarde que yo llego y llega otra a las 9 y se queda hasta otro día y así nos estamos turnando»

Es importante destacar que aunque la familia sea numerosa no todos cuidan a los padres y de igual manera las mujeres son las que cuidan.

«... los hombres el fin de semana se van de borrachos y entre semana trabajan somos 4 mujeres y nos turnamos, llegué a las 7 y se fue mi hermana la que se quedó anoche y dijo la otra yo voy a llegar como a las 4 y haber quien descansa en la noche, sino te quedas tu, una esta enferma tiene 4 días que no vienen»

Cuando se trata de cuidar al esposo y los hijos son pocos se ven en la necesidad de contratar a un cuidador formal «...llego aquí a las 6 hasta las 9 de la noche, nos sentimos cansadas pero nos turnamos para descansar tenemos contratada a una enfermera para la noche y nos vamos alternando»

La situación de enfermedad afecta a la familia, a veces de manera profunda. Cambias sus planes y trastorna su ritmo de vida, por lo que organizan las actividades de cada cuidador «...Me levanto a las 6:00, limpio mi casa y a las 12 ya estoy aquí»

Como la mayoría de las cuidadoras son amas de casa y dependen del horario es como organizan sus actividades en el hogar. «...Pues en la noche hago la comida, el día que no vengo aprovecho para lavar»

Para las cuidadoras del turno vespertino señalan que el tiempo es insuficiente para las actividades del hogar. «...le cambia a uno sus actividades y a horita la casa la tengo bueno, porque en la mañana no completo el tiempo»

El peso y fortaleza para el cuidado

Este peso del cuidado se manifiesta por alteraciones físicas por las condiciones y tiempo en que se da este.

«...mire como traigo los pies hinchados todo el día me la paso parada»

Otra manifestación se presenta en las alteraciones en el sueño «...toda la noche me la pase levantándome y así es todo el día ando flojeroza porque tengo sueño»

Una característica del cuidado informal es que no tiene horario, ni tiempo y es continuo alterando los periodos de descanso; «...no deja dormir yo me traje una toalla y un rebocito y me acosté y así me descansa el cuerpo acostada...»

Otra manifestación se relacionan con la alimentación ya que no tienen horario para comer, además influye el contexto.

«...se me quita el hambre, será el olor no se, pero no me da hambre»

Manifiestan alteraciones en el peso.

«...así come uno mas comida, es mas desórden porque aquí uno no come a sus horas y luego anda comiendo uno cualquier cosa que ni le nutre ni nada»

A pesar de su connotación negativa el cuidador, constituye un apoyo fundamental e insustituible en todos los sentidos, el enfermo necesita del cariño, cuidado y comprensión de sus familiares para sentirse seguro, para afrontar y asumir su enfermedad y en ocasiones la muerte, por lo que señalan que a veces se sienten cansados y tienen que pedir fortaleza para continuar. 
«... yo tengo mucha fe en que vamos a salir adelante y que la fuerza no es por nosotras mismas, sino que por Dios nos da la fuerza que necesitamos para hacerle frente a estas situaciones.»

Cuando es la esposa quien cuida le dedica la mayor parte del día a esta actividad y cuando les den de alta serán ellas quien lo asuman por completo

«... a noche estuve a llore y llore sola, que va a pasar, quien me va ayudar, tengo que salir adelante»

«...tengo tres hijos: dos hijas y un hijo, pero nada más dos cooperan, tienen sus trabajos, en la noche se quedan los hijos ellos se rolan, el problema va hacer cuando me lo lleve ese va hacer el problema porque yo sola voy a batallar con él»

\section{CONCLUSIONES}

Ser cuidador en un hospital reviste una serie de características que dependen de la edad del receptor del cuidado y del cuidador, así como el estado civil y parentesco. En este estudio se mostraron estas diferencias al tratar de los padres la red de cuidadores es más grande y el cuidado se comparte aunque son las mujeres quien asumen la mayor parte, pero cuando el esposo es el enfermo la esposa es quien asume la mayor parte del cuidado dependiendo del número de hijos es la ayuda y por lo general está cuida en el día.

De esta manera el cuidado en el ámbito del hospitalario se relaciona con una serie de situaciones a las que el cuidador debe de adaptarse tales como espacio y olores además a la dinámica propia de cada turno y equipo de salud. En suma con la presencia de enfermedades crónicas, los familiares no sólo participan en el tratamiento de la enfermedad. La vida familiar se ve afectada dramáticamente por la enfermedad como resultado de las funciones insatisfechas, tiempo que se consagra al tratamiento de la enfermedad, disminución de las actividades y socialización familiares, cambio de funciones, a menudo hay fatiga en la familia, el efecto en los familiar puede ser profundo, en ocasiones une a los miembros, pero en otras los aleja.

Por lo que se considera que la contribución al cuidado debería ser visible y apoyarlo para que se realice con conocimientos y de esta manera disminuya ansiedad y repercusiones en la carga del cuidado, previniendo el desgaste del cuidador porque este continuara el cuidado en casa.

\section{REFERENCIAS}

1. Zúñiga E, Vega D. Envejecimiento de la Población de México reto del siglo XXI CONAPO 2004.

2. Nigenda G, López M, Matarazzo, Ramirez C. Atención de los enfermos y discapacitados en el hogar retos para el sistema de salud mexicano. 2007; 49(004): 286-94.

3. Celma M. Cuidadores informales en el medio hospitalario. Rev Rol Enf 2001:24(8):503-11.

4. Pardo M. Familia y cuidado. Dimensiones del Cuidado. Bogota, Colombia1998;125-33.

5. Robles L. Necesitamos políticas sociales para el cuidado a largo plazo. Recuperado. 23,2005 http:wwwdebate,iteso.mx.12/articulos/ Nec.htm

6. Molina ME. Cuidar y ser cuidado: una experiencia individual. Universidad de Antioquia: 2002;142-4.

7. Rodríguez J, Zurriaga R. Estrés enfermedad y hospitalización. Granada. Escuela Andaluza de Salud Pública. 1997.

8. Robles L. Doble o triple jornada: el cuidado a enfermos crónicos. Universidad de Guadalajara, departamento de estudios del hombre, 2003;75-99.

9. Quero A. Los cuidadores no profesionales en el hospital: la mujer cuidadora. Enfermería Clínica. 2003;13(6):348-56.

10. Celma M. Cuidadores informales en el medio hospitalario. Rev Rol Enf 2001:24(8):503-11.

11. Morse J. Asuntos críticos en los métodos de investigación cualitativa. Universidad de Antioquia 2005.

12. Robles L. La invisibilidad del cuidado a los enfermos crónicos. Guadalajara Jal. Editorial Universitaria. 2007.

13. Novel G. Sistemas sociales de apoyo. En. Enfermería psicosocial: Salvart. Barcelona. 1999.81-90.

14. Quero R, Briones R, Prieto MA, Martínez N, Navarro A, Guerrero C. Los Cuidadores familiares en el hospital Universitario de traumatología y Rehabilitación de Granada. Index de Enferm. 2005; Index Enferm [online]. 2005, vol.14, n.48-49 [citado 2009-0721], pp. 14-17. Disponible en: <http://scielo.isciii.es/scielo.php? script=sci_arttext\&pid=S1132-12962005000100003\&lng=es\&nrm $=$ iso $>$. ISSN 1132-296.

15. Flores ML. La calidad de vida de los acompañantes de pacientes hospitalizados de media y larga estancia. Index de Enfermería; 2002;XI(38):124-44.

16. Andrade G, Pérez I. Apoyo de enfermería a las actividades que realizan los cuidadores informales en el ámbito hospitalario. Desarrollo Cientif Enferm 2007;15(2):52-5. 